\title{
$\mathbf{P}_{\text {ontifícia }} \mathbf{U}_{\text {niversidade }} \mathbf{C}_{\text {atólica }}$

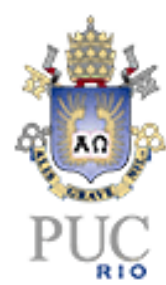

Lorena Drumond Loureiro

\section{Efeito do Número e Tamanho das Amostras Iniciais sobre o Desempenho do Gráfico de S e sobre a Estimativa da Capacidade do Processo}

\author{
Dissertação de Mestrado
}

Dissertação apresentada ao Programa de Pós-Graduação em Engenharia de Produção da PUC-Rio como requisito parcial para obtenção do título de Mestre em Engenharia de Produção.

Orientador: Eugenio Kahn Epprecht

Rio de Janeiro

Março de 2008 


\section{$\mathbf{P}_{\text {ontifícia }} \mathbf{U}_{\text {niversidade }} \mathbf{C}_{\text {atólica }}$

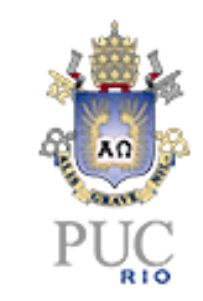

\section{Lorena Drumond Loureiro}

\section{Efeito do Número e Tamanho das Amostras Iniciais sobre o Desempenho do Gráfico de $\mathrm{S}$ e sobre a Estimativa da Capacidade do Processo}

Dissertação apresentada como requisito parcial para obtenção do grau de Mestre pelo Programa de PósGraduação em Engenharia de Produção da PUC-Rio. Aprovada pela Comissão Examinadora abaixo assinada.

Dr. Eugenio Kahn Epprecht Orientador Departamento de Engenharia de Produção - PUC-Rio

Dr. Hélio Côrtes Lopes Departamento de Matemática - PUC-Rio

Dra. Flávia Cesar Teixeira Mendes Departamento de Engenharia de Produção - PUC-Rio

Prof. José Eugenio Leal Coordenador(a) Setorial do Centro Tecnológico Científico - PUC-Rio 
Todos os direitos reservados. É proibida a reprodução total ou parcial do trabalho sem autorização da universidade, do autor e do orientador.

\section{Lorena Drumond Loureiro}

Graduou-se em Engenharia de Produção pela Universidade do Estado do Pará em 2004. Obteve o Título de Especialista em Controle Estatístico da Qualidade pela Universidade Federal do Pará em 2006.

Ficha Catalográfica

Loureiro, Lorena Drumond

Efeito do Número e Tamanho das Amostras Iniciais sobre o Desempenho do Gráfico de S e sobre a Estimativa da Capacidade do Processo/ Lorena Drumond Loureiro; Orientador: Eugenio Kahn Epprecht. - Rio de Janeiro: PUC, Departamento de Engenharia Industrial, 2008.

208 f.: il. ; $30 \mathrm{~cm}$

1. Dissertação (mestrado) - Pontifícia Universidade Católica do Rio de Janeiro, Departamento de Engenharia Industrial.

Inclui referências bibliográficas.

1. Engenharia Industrial - Teses. 2. Gráficos de Controle de S. 3. Estimação de Parâmetros. 4. Medidas de Desempenho de Gráficos de Controle. 5. Capacidade do Processo. I. Epprecht, Eugenio Kahn. II. Pontifícia Universidade Católica do Rio de Janeiro. Departamento de Engenharia Industrial. III. Título.

CDD: 658.5 
Aos meus pais, Eduardo e Conceição, às minhas irmãs Kelly, Luciana e Karine e ao meu marido, Hilbert, pelo apoio e incentivo. A toda minha família. 


\section{Agradecimentos}

A Deus que, com Sua infinita bondade, permitiu que eu chegasse até aqui.

Aos meus pais, Eduardo e Conceição, e irmãs, Kelly, Luciana e Karine, pelo incentivo e por estarem sempre presentes em minha vida.

Ao meu marido Hilbert, meu parceiro de jornada, pelo carinho e apoio incondicional.

Ao meu Professor e Orientador Eugenio Kahn Epprecht, pelos ensinamentos, pela dedicação e parceria neste trabalho, em todos os momentos.

A toda minha família, em especial à minha tia Thereza e minha prima e amiga, Juliana, que me receberam com carinho e me deram todo o apoio que precisei.

Aos amigos Laura, Isabel e Júlio, que compartilharam comigo esta etapa tão importante de nossas vidas, pelos momentos de amizade e companheirismo.

Aos professores e funcionários do Departamento de Engenharia Industrial da PUCRio, pelos ensinamentos e assistência prestada durante a realização do Mestrado.

Ao CNPq e à PUC-Rio pelo apoio financeiro concedido a mim durante o curso. 


\section{Resumo}

Loureiro, Lorena Drumond; Epprecht, Eugenio Kahn. Efeito do Número e Tamanho das Amostras Iniciais sobre o Desempenho do Gráfico de $\mathbf{S}$ e sobre a Estimativa da Capacidade do Processo. Rio de Janeiro, 2008. 208p. Dissertação de Mestrado - Departamento de Engenharia Industrial, Pontifícia Universidade Católica do Rio de Janeiro.

A probabilidade de alarme falso, $\alpha$, e o poder dos gráficos de controle de processos dependem dos seus limites de controle, que, por sua vez, dependem de estimativas dos parâmetros do processo. A análise de capacidade de processos também depende de tais estimativas. Esta dissertação apresenta inicialmente uma revisão — ao nosso conhecimento, a primeira em português — dos principais trabalhos sobre o efeito dos erros de estimação dos parâmetros do processo sobre $\alpha$. Todos os trabalhos citados buscam determinar, com base na distribuição de probabilidades das estimativas dos parâmetros do processo (parametrizada pelo número de amostras iniciais, $m$, e do tamanho delas, $n$ ) o valor esperado de $\alpha$ ou, equivalentemente, o valor esperado da distribuição marginal do número de amostras até um alarme falso. Nossa abordagem é distinta: obter (parametrizada por $n$ e $m$ ) a distribuição de $\alpha$ e seus percentis ou, equivalentemente, a distribuição do valor esperado do número de amostras até um alarme falso, de modo a fornecer orientação sobre o número de amostras iniciais a serem utilizadas antes de fixar definitivamente os valores dos limites de controle dos gráficos. A análise foi conduzida para o gráfico

de $S$. Foi analisada também a influência da estimação do desvio-padrão do processo sobre o poder do gráfico. Finalmente, foi obtida a distribuição dos erros na estimativa da capacidade do processo em função de $m$ e $n$, para fornecer orientação sobre o número de amostras necessário para garantir uma precisão especificada nessa estimativa, com um grau de confiança também especificado.

\section{Palavras-chave}

Gráficos de $S$; índices de capacidade de processos; probabilidade de alarme falso; poder; fase I; estimação de parâmetros; limites de controle; NMA; desempenho; número de amostras; tamanho de amostras; medidas de desempenho. 


\section{Abstract}

Loureiro, Lorena Dumond; Epprecht, Eugenio Kahn (Advisor). Effect of the Number and Size of the Initial Samples on the Performance of the S Chart and on the Process Capability Estimate. Rio de Janeiro, 2008. 208p. MSc. Dissertation - Departamento de Engenharia Industrial, Pontifícia Universidade Católica do Rio de Janeiro.

The false-alarm probability, $\alpha$, and the power of process control charts depend on their control limits, which, in turn, depend on process parameters estimates. Process capability analyses also depend on those estimates. This dissertation initially presents a review - to our knowledge, the first in Portuguese — of the main research articles about the effect upon $\alpha$ of the estimation errors of the process parameters. All the works reviewed aim to determine, based on the probability distribution of the process parameters estimates (which is a function of the number of initial samples, $m$, and of their size, $n$ ), the expected value of $\alpha$, or, equivalently, the expected value of the marginal distribution of the number of samples until a false alarm occurs. Our approach is different: to get (parameterized by $m$ and $n$ ) the distribution of $\alpha$ and their percentiles or, equivalently, the distribution of the expected number of samples until a false alarm, in order to provide guidance on the initial number of samples to be used before setting the chart definitive control limits. The analysis was conducted for the $S$ chart. The influence of the estimation errors on the power of the $S$ chart was also examined. Finally, the distribution function was obtained, parameterized by $m$ and $n$, of the estimation errors of the process capability index $C p$, to provide guidance on the initial number of samples required to ensure, with a specified confidence level, a specified accuracy of the estimate.

\section{Keywords}

$S$ control chart; process capability indexes; false-alarm probability; power; phase I; parameter estimation; control limits; ARL; performance; number of samples; sample size; performance measures. 


\section{Sumário}

1 Introdução 27

2 Fundamentos Conceituais e Trabalhos Precedentes 31

2.1. Fundamentos Conceituais 31

2.2. Trabalhos Precedentes 42

3 Modelo Matemático $\quad 57$

3.1. Definições Iniciais 57

3.2. Distribuição do Risco $\alpha$ e do poder $P d$ do gráfico de $S$ sob erros $\quad 59$ de estimação

3.3. Distribuição dos índices de capacidade $C p$ e $C p k$ sob erros de 63 estimação

4 Metodologia de Análise, Resultados e Discussão 65

4.1. Metodologia da Análise 1: Determinação da distribuição 66 acumulada complementar do risco $\alpha, P(\alpha>a)$, parametrizada pelo tamanho de amostra $n$ e pelo número de amostras iniciais $m$

4.1.1. Resultados e Discussão da Análise 1

4.2. Metodologia da Análise 2: Percentis do risco $\alpha$ real em função do 71 tamanho de amostras $n$ e do número de amostras $m$

4.2.1. Resultados e Discussão da Análise 2

4.3. Metodologia da Análise 3: Determinação do número mínimo de 92 amostras $m$ para cada tamanho de amostra $n$ que garante com um 
percentual de probabilidade que o risco $\alpha$ real não é maior que $\varepsilon \%$ em relação ao risco $\alpha$ nominal

4.3.1. Resultados e Discussão da Análise 3

4.4. Análise 4: O poder Pd do Gráfico de $S$

4.5 Metodologia da Análise 5: Determinação da distribuição acumulada do fator $1 / k$, de erro na estimação dos índices de capacidade $C p$ e $C p k$, parametrizada pelo tamanho de amostra $n$ e pelo número de amostras iniciais $m$

\subsubsection{Resultados e Discussão da Análise 5}

4.6. Metodologia da Análise 6: Obtenção de intervalos de confiança para $C p$ e Cpk a partir de intervalos de probabilidade para o fator $k$, em função do tamanho de amostras $n$ e do número de amostras $m$

4.6.1. Resultados e Discussão da Análise 6

4.7. Metodologia da Análise 7: Determinação do número mínimo de 125 amostras $m$ que, para cada tamanho de amostra $n$ dado, garante que $(1-\varepsilon / 100) \leq \mathrm{k} \leq(1+\varepsilon / 100)$ com probabilidade $c$, para valores de $\varepsilon$ e de $c$ especificados

\subsubsection{Resultados e Discussão da Análise 7}

5 Considerações Finais e Recomendações

6 Referências Bibliográficas

Apêndice A - Tabelas e Gráficos da Análise 1 sobre o Risco $\alpha$ do 140 Gráfico de $S$ com limites de três-sigma

Apêndice B - Tabelas e Gráficos da Análise 1 sobre o Risco $\alpha$ do Gráfico de $S$ com Limites de Probabilidade 
Apêndice C - Tabelas e Gráficos da Análise 5 sobre o fator $1 / k$, de erro na estimação dos índices de capacidade $C p$ e $C p k$ 


\section{Lista de tabelas}

Tabela 4.1 - Percentis de $95 \%$ do risco $\alpha$ real do gráfico de $S$ com limites de três-sigma em função de $n$ e $m$

Tabela 4.2 - Percentis de $90 \%$ do risco a real do gráfico de $S$ com 75 limites de três-sigma em função de $n$ e $m$

Tabela 4.3 - Percentis de $85 \%$ do risco a real do gráfico de $S$ com 77 limites de três-sigma em função de $n$ e $m$

Tabela 4.4 - Risco a do Gráfico de $S$ com limites de três-sigma 79 quando $k=1$

Tabela 4.5 - Percentis de $95 \%$ do risco a real do gráfico de $S$ com 80 limites probabilidade e $\alpha_{n o m}=0,005$, em função de $n$ e $m$

Tabela 4.6 - Percentis de $90 \%$ do risco $\alpha$ real do gráfico de $S$ com 82 limites probabilidade e $\alpha_{\text {nom }}=0,005$, em função de $n$ e $m$

Tabela 4.7 - Percentis de $85 \%$ do risco $\alpha$ real do gráfico de $S$ com limites probabilidade e $\alpha_{\text {nom }}=0,005$, em função de $n$ e $m$

Tabela 4.8 - Percentis de $95 \%$ do risco a real do gráfico de $S$ com 86 limites probabilidade e $\alpha_{\text {nom }}=0,0027$, em função de $n$ e $m$

Tabela 4.9 - Percentis de $90 \%$ do risco a real do gráfico de $S$ com 88 limites probabilidade e $\alpha_{\text {nom }}=0,0027$, em função de $n$ e $m$

Tabela 4.10 - Percentis de $85 \%$ do risco $\alpha$ real do gráfico de $S$ com 90 limites probabilidade e $\alpha_{\text {nom }}=0,0027$, em função de $n$ e $m$

Tabela 4.11 - Número mínimo de amostras iniciais $m$ em função de $n$ para o gráfico de $S$ com limites de três-sigma, com $p=5,10$ e $15 \%$ 
e $\varepsilon=10,20,30,40$ e $50 \%$

Tabela 4.12 - Número mínimo de amostras iniciais $m$ em função de $n$ 101 para $\circ$ gráfico de $S$ com limites de probabilidade, com $p=5,10$ e $15 \%, \varepsilon=10,20,30,40$ e $50 \%$, e $\alpha_{\text {nom }}=0,005$

Tabela 4.13 - Número mínimo de amostras iniciais $m$ em função de $n$ 104 para $\circ$ gráfico de $S$ com limites de probabilidade, com $p=5,10$ e $15 \%, \varepsilon=10,20,30,40$ e $50 \%$, e $\alpha_{\text {nom }}=0,0027$

Tabela $4.14 \mathrm{a}$ - Intervalos de $80 \%$ de probabilidade para o fator de 116 erro $k$ para diferentes $n$ e $m=10,15,20,25,30,40$ e 50: limites $k_{\text {inf }}$ e $k_{\text {sup }}$ para construção de IC de $80 \%$ para $C p$ e $C p k$

Tabela $4.14 \mathrm{~b}$ - Intervalos de $80 \%$ de probabilidade para o fator de erro $k$ para diferentes $n$ e $m=60,70,80,90,100,150$ e 200: limites $k_{\text {inf }}$ e $k_{\text {sup }}$ para construção de IC de $80 \%$ para Cp e Cpk

Tabela $4.15 a$ - Intervalos de $90 \%$ de probabilidade para o fator de erro $k$ para diferentes $n$ e $m=10,15,20,25,30,40$ e 50: limites $k_{\text {inf }} \mathrm{e}$ $k_{\text {sup }}$ para construção de IC de $90 \%$ para $C p$ e Cpk

Tabela $4.15 b$ - Intervalos de $90 \%$ de probabilidade para o fator de 120 erro $k$ para diferentes $n$ e $m=60,70,80,90,100,150$ e 200: limites $k_{\text {inf }}$ e $k_{\text {sup }}$ para construção de IC de $90 \%$ para Cp e Cpk

Tabela 4.16 a - Intervalos de $95 \%$ de probabilidade para o fator de 122 erro $k$ para diferentes $n$ e $m=10,15,20,25,30,40$ e 50: limites $k_{\text {inf }} \mathrm{e}$ $k_{\text {sup }}$ para construção de IC de $95 \%$ para $C p$ e Cpk

Tabela $4.16 \mathrm{~b}$ - Intervalos de $95 \%$ de probabilidade para o fator de 123 erro $k$ para diferentes $n$ e $m=60,70,80,90,100,150$ e 200: limites $k_{\text {inf }}$ e $k_{\text {sup }}$ para construção de IC de $95 \%$ para Cp e Cpk 
Tabela 4.17 - Número mínimo de amostras iniciais $m$ em função de $n$ para $c=80,90$ e $95 \%$ e $\varepsilon=5,10,20$ e $30 \%$

\section{Tabelas do Apêndice A}

Tabela A.1 - Distribuição Acumulada Complementar $P(\alpha>$ a), para $n=2,3$ e 4

Tabela A.2 - Distribuição Acumulada Complementar $P(\alpha>a)$, para 145 $n=5,6$ e 8

Tabela A.3 - Distribuição Acumulada Complementar $P(\alpha>$ a), para 149 $n=10,12$ e 15

Tabela A.4 - Distribuição Acumulada Complementar $P(\alpha>$ a), para 153 $n=20,25$ e 30

Tabelas do Apêndice B

Tabela B.1 - Distribuição Acumulada Complementar $P(\alpha>a)$, para 158 $n=2,3$ e 4 e $\alpha_{\text {nom }}=0,0050$

Tabela B.2 - Distribuição Acumulada Complementar $P(\alpha>$ a) para 162 $n=5,6$ e 8 e $\alpha_{\text {nom }}=0,0050$

Tabela B.3 - Distribuição Acumulada Complementar $P(\alpha>a)$ para 166 $n=10,12$ e 15 e $\alpha_{\text {nom }}=0,0050$

Tabela B.4 - Distribuição Acumulada Complementar P $(\alpha>$ a) para $n$ 170 $=20,25$ e 30 e $\alpha_{\text {nom }}=0,0050$

Tabela B.5 - Distribuição Acumulada Complementar P $(\alpha>$ a) para 174 $n=2,3$ e 4 e $\alpha_{\text {nom }}=0,0027$

Tabela B.6 - Distribuição Acumulada Complementar P $(\alpha>$ a) para 
$n=5,6$ e 8 e $\alpha_{\text {nom }}=0,0027$

Tabela B.7 - Distribuição Acumulada Complementar P $(\alpha>$ a) para

$n=10,12$ e 15 e $\alpha_{\text {nom }}=0,0027$

Tabela B.8 - Distribuição Acumulada Complementar $P(\alpha>$ a) para

$n=20,25$ e 30 e $\alpha_{\text {nom }}=0,0027$

Tabelas do Apêndice C

Tabela C.1 - Distribuição acumulada do fator $1 / k$, de erro na estimação dos índices de capacidade $C p$ e $C p k$, para $n=2$ e 3

Tabela C.2 - Distribuição acumulada do fator $1 / k$, de erro na 194 estimação dos índices de capacidade $C p$ e $C p k$, para $n=4$ e 5

Tabela C.3 - Distribuição acumulada do fator $1 / k$, de erro na estimação dos índices de capacidade $C p$ e $C p k$, para $n=6$ e 8

Tabela C.4 - Distribuição acumulada do fator $1 / k$, de erro na 200 estimação dos índices de capacidade $C p$ e $C p k$, para $n=10$ e 12

Tabela C.5 - Distribuição acumulada do fator $1 / k$, de erro na 203 estimação dos índices de capacidade $C p$ e $C p k$, para $n=15$ e 20

Tabela C.6 - Distribuição acumulada do fator $1 / k$, de erro na 206 estimação dos índices de capacidade $C p$ e $C p k$, para $n=25$ e 30 


\section{Lista de figuras}

Figura 4.1 - Gráfico dos percentis de $95 \%$ do risco $\alpha$ real do gráfico de $S$ com limites de três-sigma em função de $n$ e $m$

Figura 4.2 - Gráfico dos percentis de $90 \%$ do risco a real do gráfico 76 de $S$ com limites de três-sigma em função de $n$ e $m$

Figura 4.3 - Gráfico dos percentis de $85 \%$ do risco $\alpha$ real do gráfico de $S$ com limites de três-sigma em função de $n$ e $m$

Figura 4.4 - Gráfico dos percentis de $95 \%$ do risco $\alpha$ real do gráfico de $S$ com limites probabilidade e $\alpha_{\text {nom }}=0,0050$, em função de $n$ e $m$

Figura 4.5 - Gráfico dos percentis de $90 \%$ do risco a real do gráfico de $S$ com limites probabilidade e $\alpha_{n o m}=0,0050$, em função de $n$ e $m$

Figura 4.6 - Gráfico dos percentis de $85 \%$ do risco $\alpha$ real do gráfico de $S$ com limites probabilidade e $\alpha_{\text {nom }}=0,0050$, em função de $n$ e $m$

Figura 4.7 - Gráfico dos percentis de $95 \%$ do risco a real do gráfico de $S$ com limites probabilidade e $\alpha_{n o m}=0,0027$, em função de $n$ e $m$

Figura 4.8 - Gráfico dos percentis de $90 \%$ do risco a real do gráfico de $S$ com limites probabilidade e $\alpha_{n o m}=0,0027$, em função de $n$ e $m$

Figura 4.9 - Gráfico dos percentis de $85 \%$ do risco $\alpha$ real do gráfico de $\mathrm{S}$ com limites probabilidade e $\alpha_{n o m}=0,0027$, em função de $n$ e $m$

Figura 4.10 - Gráfico do número mínimo de amostras iniciais $m$ em função de $n$ para o gráfico de $S$ com limites de três-sigma, com $\varepsilon=10,20,30,40$ e $50 \%$ e $p=5 \%$ 
Figura 4.11 - Gráfico do número mínimo de amostras iniciais $m$ em

função de $n$ para o gráfico de $S$ com limites de três-sigma, com $\varepsilon=10,20,30,40$ e $50 \%$ e $p=10 \%$

Figura 4.12 - Gráfico do número mínimo de amostras iniciais $m$ em

função de $n$ para o gráfico de $S$ com limites de três-sigma, com $\varepsilon=10,20,30,40$ e $50 \%$ e $p=15 \%$

Figura 4.13 - Gráfico do número mínimo de amostras iniciais $m$ em 102 função de $n$ para o gráfico de $S$ com limites de probabilidade, com $\varepsilon=10,20,30,40$ e $50 \%, p=5 \%$ e $\alpha_{\text {nom }}=0,005$

Figura 4.14 - Gráfico do número mínimo de amostras inicial $m$ em 102 função de $n$ para o gráfico de $S$ com limites de probabilidade, com $\varepsilon=10,20,30,40$ e $50 \%, p=10 \%$ e $\alpha_{\text {nom }}=0,005$

Figura 4.15 - Gráfico do número mínimo de amostras inicial $\mathrm{m} \mathrm{em}$ 103 função de $n$ para o gráfico de $S$ com limites de probabilidade, com $\varepsilon=10,20,30,40$ e $50 \%, p=15 \%$ e $\alpha_{\text {nom }}=0,005$

Figura 4.16 - Gráfico do número mínimo de amostras iniciais $m$ em 105 função de $n$ para o gráfico de $S$ com limites de probabilidade, com $\varepsilon=10,20,30,40$ e $50 \%, p=5 \%$ e $\alpha_{\text {nom }}=0,0027$

Figura 4.17 - Gráfico do número mínimo de amostras iniciais $m$ em 105 função de $n$ para o gráfico de $S$ com limites de probabilidade, com $\varepsilon=10,20,30,40$ e $50 \%, p=10 \%$ e $\alpha_{\text {nom }}=0,0027$

Figura 4.18 - Gráfico do número mínimo de amostras iniciais $m$ em 106 função de $n$ para o gráfico de $S$ com limites de probabilidade, com $\varepsilon=10,20,30,40$ e $50 \%, p=15 \%$ e $\alpha_{\text {nom }}=0,0027$

Figura 4.19 - Gráfico dos Intervalos de $80 \%$ de probabilidade para o fator de erro $k$ para diferentes $n$ e $m$ : limites $k_{\text {inf }}$ e $k_{\text {sup }}$ para 
construção de IC de $80 \%$ para $C p$ e $C p k$

Figura 4.20 - Gráfico dos Intervalos de $90 \%$ de probabilidade para o 121 fator de erro $k$ para diferentes $n$ e $m$ : limites $k_{\text {inf }}$ e $k_{\text {sup }}$ para construção de IC de $90 \%$ para Cp e Cpk

Figura 4.21 - Gráfico dos Intervalos de $95 \%$ de probabilidade para o 124 fator de erro $k$ para diferentes $n$ e $m$ : limites $k_{\text {inf }}$ e $k_{\text {sup }}$ para construção de IC de 95\% para Cp e Cpk

Figura 4.22 - Gráfico do número mínimo de amostras iniciais $m$ em 129 função de $n$ para $c=80 \%$ e $\varepsilon=5,10,20$ e $30 \%$

Figura 4.23 - Gráfico do número mínimo de amostras iniciais $m$ em 129 função de $n$ para $c=90 \%$ e $\varepsilon=5,10,20$ e $30 \%$

Figura 4.24 - Gráfico do número mínimo de amostras iniciais $m$ em 130 função de $n$ para $c=95 \%$ e $\varepsilon=5,10,20$ e $30 \%$

\section{Figuras do Apêndice A}

Figura A.1 - Gráfico da distribuição acumulada complementar 142 $P(\alpha>a)$, para $n=2$

Figura A.2 - Gráfico da distribuição acumulada complementar 143 $P(\alpha>a)$, para $n=3$

Figura A.3 - Gráfico da distribuição acumulada complementar 144 $P(\alpha>a)$, para $n=4$

Figura A.4 - Gráfico da distribuição acumulada complementar 146 $P(\alpha>a)$, para $n=5$

Figura A.5 - Gráfico da distribuição acumulada complementar $P(\alpha>a)$, para $n=6$ 
Figura A.6 - Gráfico da distribuição acumulada complementar $P(\alpha>a)$, para $n=8$

Figura A.7 - Gráfico da distribuição acumulada complementar $P(\alpha>a)$, para $n=10$

Figura A.8 - Gráfico da distribuição acumulada complementar $P(\alpha>a)$, para $n=12$

Figura A.9 - Gráfico da distribuição acumulada complementar $P(\alpha>a)$, para $n=15$

Figura A.10 - Gráfico da distribuição acumulada complementar 154 $P(\alpha>a)$, para $n=20$

Figura A.11 - Gráfico da distribuição acumulada complementar $P(\alpha>a)$, para $n=25$

Figura A.12 - Gráfico da distribuição acumulada complementar 156 $P(\alpha>a)$, para $n=30$

Figuras do Apêndice B

Figura B.1 - Gráfico da distribuição acumulada complementar 159 $P(\alpha>a)$, para $n=2$ e $\alpha_{\text {nom }}=0,005$

Figura B.2 - Gráfico da distribuição acumulada complementar 160 $P(\alpha>a)$, para $n=3$ e $\alpha_{n o m}=0,005$

Figura B.3 - Gráfico da distribuição acumulada complementar 161 $P(\alpha>a)$, para $n=4$ e $\alpha_{\text {nom }}=0,005$

Figura B.4 - Gráfico da distribuição acumulada complementar 163 $P(\alpha>a)$, para $n=5$ e $\alpha_{\text {nom }}=0,005$ 
Figura B.5 - Gráfico da distribuição acumulada complementar $P(\alpha>a)$, para $n=6$ e $\alpha_{\text {nom }}=0,005$

Figura B.6 - Gráfico da distribuição acumulada complementar $P(\alpha>a)$, para $n=8$ e $\alpha_{\text {nom }}=0,005$

Figura B.7 - Gráfico da distribuição acumulada complementar $P(\alpha>a)$, para $n=10$ e $\alpha_{n o m}=0,005$

Figura B.8 - Gráfico da distribuição acumulada complementar 168 $P(\alpha>a)$, para $n=12$ e $\alpha_{\text {nom }}=0,005$

Figura B.9 - Gráfico da distribuição acumulada complementar 169 $P(\alpha>a)$, para $n=15$ e $\alpha_{\text {nom }}=0,005$

Figura B.10 - Gráfico da distribuição acumulada complementar 171 $P(\alpha>a)$, para $n=20$ e $\alpha_{\text {nom }}=0,005$

Figura B.11 - Gráfico da distribuição acumulada complementar 172 $P(\alpha>a)$, para $n=25$ e $\alpha_{\text {nom }}=0,005$

Figura B.12 - Gráfico da distribuição acumulada complementar 173 $P(\alpha>a)$, para $n=30$ e $\alpha_{\text {nom }}=0,005$

Figura B.13 - Gráfico da distribuição acumulada complementar 175 $P(\alpha>a)$, para $n=2$ e $\alpha_{\text {nom }}=0,0027$

Figura B.14 - Gráfico da distribuição acumulada complementar 176 $P(\alpha>a)$, para $n=3$ e $\alpha_{\text {nom }}=0,0027$

Figura B.15 - Gráfico da distribuição acumulada complementar $P(\alpha>a)$, para $n=4$ e $\alpha_{\text {nom }}=0,0027$

Figura B.16 - Gráfico da distribuição acumulada complementar 179 $P(\alpha>a)$, para $n=5$ e $\alpha_{\text {nom }}=0,0027$ 
Figura B.17 - Gráfico da distribuição acumulada complementar $P(\alpha>a)$, para $n=6$ e $\alpha_{\text {nom }}=0,0027$

Figura B.18 - Gráfico da distribuição acumulada complementar $P(\alpha>a)$, para $n=8$ e $\alpha_{\text {nom }}=0,0027$

Figura B.19 - Gráfico da distribuição acumulada complementar 183 $P(\alpha>a)$, para $n=10$ e $\alpha_{\text {nom }}=0,0027$

Figura B.20 - Gráfico da distribuição acumulada complementar $P(\alpha>a)$, para $n=12$ e $\alpha_{\text {nom }}=0,0027$

Figura B.21 - Gráfico da distribuição acumulada complementar 185 $P(\alpha>a)$, para $n=15$ e $\alpha_{\text {nom }}=0,0027$

Figura B.22 - Gráfico da distribuição acumulada complementar 187 $P(\alpha>a)$, para $n=20$ e $\alpha_{\text {nom }}=0,0027$

Figura B.23 - Gráfico da distribuição acumulada complementar 188 $P(\alpha>a)$, para $n=25$ e $\alpha_{\text {nom }}=0,0027$

Figura B.24 - Gráfico da distribuição acumulada complementar 189 $P(\alpha>a)$, para $n=30$ e $\alpha_{\text {nom }}=0,0027$

\section{Figuras do apêndice C}

Figura C.1 - Gráfico da Distribuição Acumulada do Fator de Erro 1/k, 192 para $n=2$

Figura C.2 - Gráfico da Distribuição Acumulada do Fator de Erro 1/k, 193 para $n=3$

Figura C.3 - Gráfico da Distribuição Acumulada do Fator de Erro 1/k, 195 para $n=4$ 
Figura C.4 - Gráfico da Distribuição Acumulada do Fator de Erro 1/k,

para $n=5$

Figura C.5 - Gráfico da Distribuição Acumulada do Fator de Erro 1/k,

para $n=6$

Figura C.6 - Gráfico da Distribuição Acumulada do Fator de Erro 1/k,

para $n=8$

Figura C.7 - Gráfico da Distribuição Acumulada do Fator de Erro 1/k,

para $n=10$

Figura C.8 - Gráfico da Distribuição Acumulada do Fator de Erro 1/k, 202 para $n=12$

Figura C.9 - Gráfico da Distribuição Acumulada do Fator de Erro 1/k, 204 para $n=15$

Figura C.10 - Gráfico da Distribuição Acumulada do Fator de Erro 205 $1 / k$, para $n=20$

Figura C.11 - Gráfico da Distribuição Acumulada do Fator de Erro 207 $1 / k$, para $n=25$

Figura C.12 - Gráfico da Distribuição Acumulada do Fator de Erro 208 $1 / k$, para $n=30$ 


\section{Lista de abreviaturas e símbolos}

$1 / k$

$a$

$A_{2}$

$b$

C

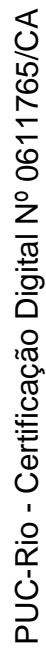

$C_{4}$

CEP

CEQ

$C p$

$C p_{a}$

Cpk

Fator de erro na estimação de $C p$ ou $C p k$

valores máximos para o risco $\alpha$ do gráfico de controle

Constante tabelada em função do tamanho de amostra $n$

Razão entre o desvio-padrão para o processo fora de controle e o desvio-padrão-alvo

Percentual de confiança do intervalo de probabilidade para $k$

Constante tabelada em função do tamanho de amostra $n$

Controle estatístico do processo

Controle estatístico da qualidade

Índice de capacidade

Índice de capacidade $C p$ aparente

Índice de capacidade

Cpka

Índice de capacidade $C p k_{a}$ aparente

Cpm Índice de capacidade

$D_{3}$

$D_{4}$

$F_{1 / k}$

Densidade de probabilidade acumulada para $1 / \mathrm{k}$

Fase I

$1^{\text {a }}$ fase dos gráficos de controle

Fase II

$2^{\text {a }}$ fase dos gráficos de controle

$F_{k}$

Densidade de probabilidade acumulada para $k$

$F_{\alpha} a$ 


\begin{tabular}{|c|c|}
\hline$h$ & Intervalo de tempo entre amostras \\
\hline$h_{\gamma, m, k, v}^{\prime}$ & $\begin{array}{l}\text { Valores críticos da distribuição normal } k \text {-variada das } \\
\text { diferenças } \bar{X}_{i}-\overline{\bar{X}} \text {. }\end{array}$ \\
\hline$H_{0}$ & Hipótese inicial \\
\hline$H_{1}$ & Hipótese alternativa \\
\hline$k$ & Fator de erro na estimação do desvio-padrão do processo \\
\hline$k_{a}$ & $\begin{array}{l}\text { valor de } k \text { que corresponde a um valor para o risco } \alpha \\
\text { igual a } a \text {. }\end{array}$ \\
\hline$k_{\text {inf }}$ & Limite inferior do intervalo de probabilidade para $k$ \\
\hline$k_{p}$ & Valor de $k$ correspondente a $p$ \\
\hline$k_{\text {sup }}$ & Limite superior do intervalo de probabilidade para $k$ \\
\hline$L \hat{C} L$ & Limite inferior de controle estimado na Fase I \\
\hline$L I C$ & Limite inferior de controle \\
\hline$L I C_{S}$ & $\begin{array}{l}\text { Limite inferior do gráfico de controle do desvio-padrão } \\
\text { amostral } S\end{array}$ \\
\hline LIE & Limite inferior de especificação \\
\hline LSC & Limite superior de controle \\
\hline$L S C_{S}$ & $\begin{array}{l}\text { Limite superior do gráfico de controle do desvio-padrão } \\
\text { amostral } S\end{array}$ \\
\hline LSE & Limite superior de especificação \\
\hline$m$ & Número de amostras \\
\hline$n$ & Tamanho da amostra \\
\hline $\begin{array}{l}N M A \text { ou } N M A_{1} \\
\text { ou } A R L\end{array}$ & Número médio de amostras até o sinal \\
\hline
\end{tabular}

$N M A_{0}$ ou $A R L_{0} \quad$ Número médio de amostras até um alarme falso 


\begin{tabular}{|c|c|}
\hline$p$ & Probabilidade de o risco a ultrapassar o valor máximo a \\
\hline$P d$ & Probabilidade de detecção \\
\hline$R$ & Amplitude amostral \\
\hline$R L$ & Número de amostras até o sinal \\
\hline$S$ & Desvio-padrão amostral \\
\hline $\bar{S}$ & média dos $m$ desvios-padrão $S_{j}, \mathrm{j}=1,2, \ldots, m$ \\
\hline$S^{2}$ & Variância amostral \\
\hline$S D R L$ & desvio-padrão do $R L$ \\
\hline TES & Tempo esperado até o sinal \\
\hline TMAF & Tempo médio até um alarme falso \\
\hline$U \hat{C} L$ & Limite superior de controle estimado na Fase I \\
\hline$V$ & $\begin{array}{l}\text { número de amostras até que o primeiro sinal ocorra para } \\
\text { o caso em que } \sigma \text { é conhecido }\end{array}$ \\
\hline$v, w$ & Valores possíveis das estimativas dos parâmetros \\
\hline$w$ & $\begin{array}{l}\text { número de amostras até que o primeiro sinal ocorra para } \\
\text { o caso em que } \sigma \text { é estimado }\end{array}$ \\
\hline$x$ & variável aleatória \\
\hline$\overline{\mathrm{X}}$ & Média amostral \\
\hline$\overline{\bar{X}}$ & Valor médio das médias das amostras \\
\hline$\chi_{n-1, \alpha}^{2}$ & $\begin{array}{l}\text { quantil de }(1-\alpha) \text { da variável qui-quadrado com } n-1 \\
\text { graus de liberdade }\end{array}$ \\
\hline$\chi_{n-1, \alpha_{\text {real }}}^{2}$ & $\begin{array}{l}\text { Valor qui-quadrado real correspondente ao limite superior } \\
\text { de controle do gráfico de } S \text {, dado que existe um erro na } \\
\text { estimação e/ou deslocamento do desvio-padrão do } \\
\text { processo }\end{array}$ \\
\hline
\end{tabular}


$\chi_{n-1, \alpha_{n o m}}^{2}$

$z$

$\alpha$

$\alpha_{\text {nom }}$

$\alpha_{\text {real }}$

$\beta$

$\Delta$

$\mu$

$\mu_{k}$

$\sigma$

$\sigma_{0}$

$\hat{\sigma}_{0}$

$\sigma_{1}$

$\sigma^{2}$

$\sigma_{k}$

$\mathcal{E}$

$\gamma$

$\gamma^{\prime}$

Valor qui-quadrado nominal correspondente ao limite superior de controle do gráfico de $S$ quando o desviopadrão do processo é conhecido

valores mínimos para o poder do gráfico de controle

Probabilidade de alarme falso ou erro do tipo I

Probabilidade de alarme falso ou erro do tipo I nominal

Probabilidade de alarme falso ou erro do tipo I dado que existe um erro na estimação e/ou deslocamento do desvio-padrão do processo

Probabilidade de não-detecção ou erro do tipo II

Semi-largura do intervalo de confiança para Cp e Cpk

Média do processo

Média de $k$

Desvio-padrão do processo

Desvio-padrão do processo em controle

Estimativa do desvio-padrão do processo $\sigma_{0}$

Desvio-padrão do processo após ter sofrido um aumento (deslocamento)

Variância populacional

Desvio-padrão de $k$

Diferença percentual de $\alpha_{\text {real }}$ em relação ao $\alpha_{\text {nom }}$ e semilargura do intervalo de probabilidade para $k$

Razão entre o desvio padrão do processo após um aumento $\left(\sigma_{1}\right)$ e o desvio-padrão do processo em controle

Fator $\gamma$ aparente 


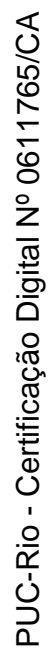

"Não se pode melhorar o que não se mede"

Autor desconhecido 